\title{
MITRAGYNA SPECIOSA-INDUCED HEPATOTOXICITY-TREATED EFFECTIVELY BY PIPER BETLE: SCOPE AS A FUTURE ANTIDOTE
}

\author{
HASZIANALIZA HASLAN ${ }^{1,2}$, FARIHAH HAJI SUHAIMI ${ }^{1}$, SRIJIT DAS ${ }^{1 *}$ \\ ${ }^{1}$ Department of Anatomy, Universiti Kebangsaan Malaysia Medical Centre, Jalan Yaacob Latif, Bandar Tun Razak, 56000 Cheras, Kuala \\ Lumpur, Malaysia. ${ }^{2}$ Anatomy Unit, Faculty of Medicine, Universiti Teknologi Mara, Sungai Buloh Campus, Jalan Hospital, 47000 Sungai \\ Buloh, Selangor Darul Ehsan. Email: Drsrijit@gmail.com \\ Received: 06 October 2017, Revised and Accepted: 22 November 2017
}

ABSTRACT

Objective: Consumption of Mitragyna speciosa (MS) leads to various toxicities including hepatotoxicity. Piper betle (PB) is a herb that possesses various therapeutic properties. The aim of the present study was to examine the protective effect of PB methanol extract (PBME) on MS-induced hepatotoxicity which could pave the way for any future antidote.

Methods: Twenty-four male Sprague-Dawley rats were randomized into control and experimental groups. The control group was further divided into the negative (G-T80) and positive (G-PB) control groups. The G-T80 group ( $\mathrm{n}=6)$ received oral gavage of the vehicle, 15\% Tween 80 . The G-PB group (n=6) received PBME $200 \mathrm{mg} / \mathrm{kg} /$ day, orally. The experimental group was divided into two groups, i.e., The G-MS and G-MS (PB) groups. The G-MS group $(\mathrm{n}=6)$ received only MS methanol extract (MSME) $500 \mathrm{mg} / \mathrm{kg} /$ day, while the G-MS (PB) group (n=6) received MSME with concomitant treatment with PBME.

Results: Histopathology examination of the G-T80 and G-PB groups showed normal histology of the liver. The G-MS group showed liver injury features such as microvesicular steatosis, ballooning degeneration, acidophilic bodies, scattered focal necrosis, fibrous portal expansion, bridging fibrosis, sinusoidal congestion, and dilatation. These features were fewer in the G-MS (PB) group which received concomitant treatment with PBME.

Conclusion: Administration of PBME exerted a protective effect against MS-induced hepatotoxicity. Future clinical trials using PB as an antidote may help in combating MS-induced hepatoxicity.

Keywords: Mitragyna speciosa, Piper betle, Liver injury, Methanol extract, Microvesicular steatosis, Fibrosis.

(C) 2018 The Authors. Published by Innovare Academic Sciences Pvt Ltd. This is an open access article under the CC BY license (http://creativecommons. org/licenses/by/4. 0/) DOI: http://dx.doi.org/10.22159/ajpcr.2018.v11i3.22958

\section{INTRODUCTION}

Mitragyna speciosa (MS) is a native plant of South-East Asia and it is also known as "ketum" in Malaysia or "kratom" in Thailand. It is a tropical, evergreen plant which belongs to the Rubiaceae family. Its height may reach up to 25 feet with a diameter of $2-3$ feet [1]. Conventionally, it has been used in this region to treat various ailments including pain, diarrhea, fever, worm infestation, cough, and increase sexual performance $[2,3]$.

Recent evidence shows an increase in toxicity associated with MS usage $[2,3]$. MS has been misused by locals in many countries. Liver being the main organ in detoxification process is the most vulnerable organ to any toxic injury [4]. Several case reports and experimental studies reported liver injury associated with MS consumption. Intrahepatic cholestasis was diagnosed in a 25-year-old man following consumption of MS with rapid dosage increment [5]. Cholestatic hepatitis was reported to develop in a 58-year-old man following 3 months' consumption of MS [6]. Numerous experimental studies reported the development of liver injury following administration of MS extract in acute and subchronic duration [7-10]. Hence, there is a need to look for a potential antidote which can inhibit hepatotoxicity caused by MS.

Piper betle (PB) is herbal plant belongs to Piperaceae family. It is a perennial climber which is commonly found in many Asian countries. Numerous scientific studies reported therapeutic properties of PB $[11,12]$. PB was reported to exhibit hepatoprotective effect against various toxic insults including cadmium, carbon tetrachloride, ethanol, and D-galactosamine [13-16]. Hence, in the present study, we aimed to observe the effect of PB in hepatotoxicity caused by MS.

\section{MATERIALS AND METHODS}

\section{Ethical clearance}

The animal studies were performed after receiving approval from the Institutional Animal Care and Use Committee in Universiti Kebangsaan Malaysia.

\section{Plant materials}

M. speciosa and PB were bought from Hilleaf resources, Sungai Buloh, Selangor. The leaves were identified by Herbarium, Faculty of Science and Technology, Universiti Kebangsaan Malaysia (UKM), Bangi, Malaysia, and conserved there with specimen voucher numbers for MS and PB as UKMB 30028 and UKMB 40238, respectively.

\section{Methanol crude extracts preparation}

M. speciosa leaves were washed thoroughly, and twigs were removed to ensure that only the leaves were left out. The leaves were dried under sunlight. The dried leaves were grounded into finer pieces. A total of $250 \mathrm{~g}$ of grounded leaves were exhaustively Soxhlet extracted with 2.5 L methanol, producing dark-brown extract solution. The methanol was evaporated from the extract solution using rotary evaporator (Heidolph, German) and vacuum pump (Buchi, Switzerland) producing $33 \mathrm{~g}$ of black paste of crude MS methanol extract (MSME). PB leaves underwent similar process as per description for MS leaves. Thus, $250 \mathrm{~g}$ of PB dried leaves yielded $30 \mathrm{~g}$ of dark-green paste of crude PB methanol extract (PBME).

Animals

Twenty-four ( $\mathrm{n}=24)$ male Sprague-Dawley rats $(190 \pm 10 \mathrm{~g})$ were bought from the Laboratory Animal Resource Unit, UKM. Each rat 
was housed in individual plastic cage and was maintained under standard environmental condition (12 h light/dark cycle) with a room temperature of $27^{\circ} \mathrm{C} \pm 2^{\circ} \mathrm{C}$. The rats were fed with standard rat pellets and water ad libitum and were acclimatized for 1 week before being subjected to the experimental procedures.

\section{Study design}

The rats $(n=24)$ were randomly divided into two groups, i.e., control and experimental groups. Control group was further subdivided into two groups: (i) Negative (G-T80) and (ii) positive (G-PB) control groups with each group consisting of six rats. The G-T80 group received the vehicle, 15\% Tween 80 administered through oral gavage for 28 days. The G-PB group received $200 \mathrm{mg} / \mathrm{kg} /$ day PBME dissolved in $15 \%$ Tween 80 administered through oral gavage for 28 days. The experimental group was subdivided into two, i.e., G-MS and G-MS (PB) groups with each group consisting of six rats. The experimental groups received $500 \mathrm{mg} / \mathrm{kg} /$ day MSME dissolved in 15\% Tween 80 orally for 28 days. The G-MS group received only MSME, and no treatment was given. The G-MS (PB) group received MSME and concomitant treatment with PBME $200 \mathrm{mg} / \mathrm{kg}$ for 28 days. The animal experiments were performed as per earlier published study [9].

\section{Histopathological examination}

At the end of experiment, the rats were sacrificed and the liver was harvested. The liver was washed with $0.9 \%$ normal saline to remove excess blood. The liver tissue was fixed in $10 \%$ formalin at room temperature. The fixed tissues underwent dehydration with a series of increasing concentration of alcohol solutions, followed by clearing with xylene. The tissues were embedded in paraffin wax, and $5 \mu \mathrm{m}$ tissues were section and stained with hematoxylin and eosin, reticulin, and Masson's trichrome. The slide was examined and the image was captured using image analyzer DMRXA2 (Leica, German).

\section{RESULTS}

\section{G-T80 group}

The G-T80 group showed normal histology of the liver (Fig. 1). Liver parenchyma mainly comprised hepatocytes that were polyhedral in shaped with euchromatic nucleus. Hepatocytes formed the hepatic plates which converged toward the central vein (CV). Hepatic plates were mostly formed by single layer of hepatocytes. Under reticulin staining, the converging pattern of hepatic plates could be clearly appreciated (Fig. 2). Liver sinusoids were located in between the anastomosing hepatic plates. Portal tract (PT) consisted of portal veins tributaries, branches of hepatic artery, and bile duct. These structures were bounded together by connective tissue. Under Masson's trichrome staining, collagen around the PT could be clearly appreciated and the collagen was stained blue (Fig. 3)

\section{G-PB group}

Histopathology assessment of the G-PB group showed that the liver tissue was comparable to the G-T80 group which indicated normal histology of the liver (Fig. 1). Liver lobules had CV in the center with portal triad at its periphery (Fig. 2). Hepatocytes were polyhedral and formed anastomosing hepatic plates. No fibrotic change was observed in the G-PB group (Fig. 3).

\section{G-MS group}

Liver tissue of the G-MS (PB) group showed liver injury features. There was severe sinusoidal congestion and dilatation with disrupted $\mathrm{CV}$ (Fig. 1). There was scattered focal necrosis with inflammatory cell infiltration. Under reticulin stain, there were areas of "drop out" lesion which were devoid of parenchyma (Fig. 2). Acidophilic bodies were observed and were characterized by shrunken cells with eosinophilic cytoplasm that was irregularly shaped and contained pyknotic nucleus (Fig. 4). Few hepatocytes developed ballooning degeneration, characterized by swollen and paler hepatocytes (Fig. 4). Microvesicular steatosis characterized by several tiny lipid droplets was present in some hepatocytes (Fig. 4). It appeared as if there was discontinuation

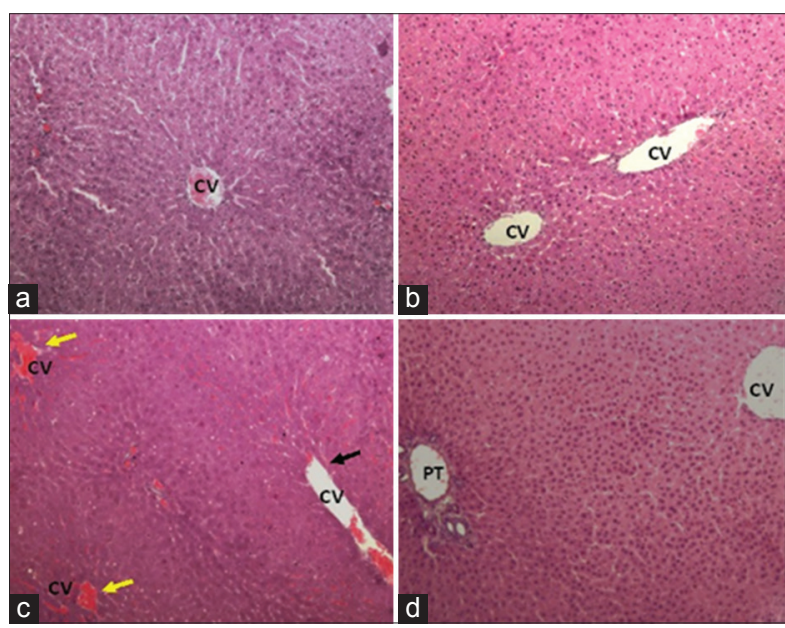

Fig. 1: Histology of the liver. (a) The G-T80 and (b) G-Piper betle $(\mathrm{PB})$ groups showed normal histology of the liver. (c) The G-Mitragyna speciosa (MS) group showed sinusoidal congestion (yellow arrow) and dilatation (black arrow) with disruption of the central veins (CV). (d) The G-MS PB group showed normal structure of $\mathrm{CV}$ and portal tract. (Hematoxylin and eosin stain; original magnification, $\times \mathbf{1 0}$ )

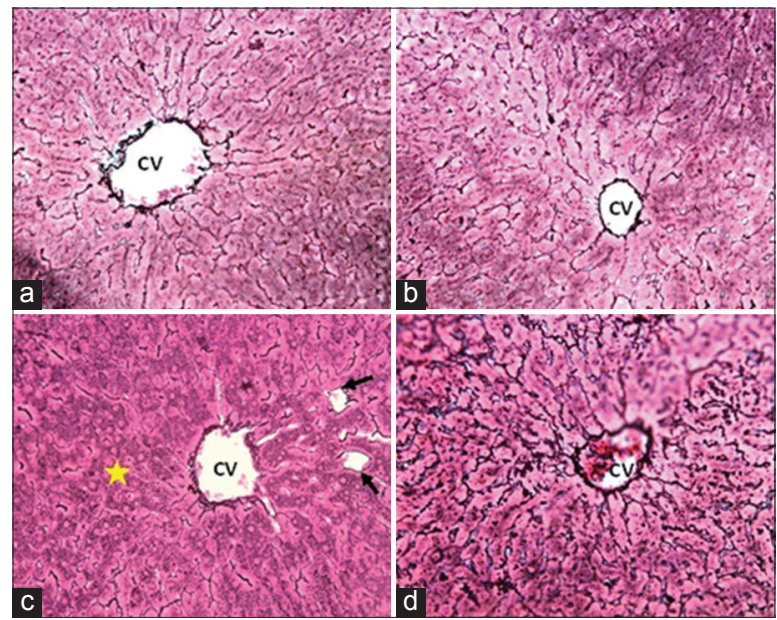

Fig. 2: Centrilobular area of the liver. (a) The G-T80 and (b) G-Piper betle (PB) groups showed reticulin fibers lined the centra vein (CV), hepatic plates, and sinusoids. (c) The G-Mitragyna speciosa (MS) group showed areas with discontinuation of reticulin fibers (yellow star). Focal necrosis (black arrows) appeared as "drop out" areas within liver parenchyma. (d) The

G-MS PB group showed CV lined by reticulin fibers and the hepatic plates showed converging pattern. (Reticulin stain, $\times 20$ )

of reticulum fiber when viewed under reticulin stain (Fig. 2). Masson's trichrome staining showed few areas of fibrous portal expansion and bridging fibrosis (Fig. 3). The liver tissue showed features suggestive of hepatotoxicity.

\section{G-MS (PB) group}

Normal liver architecture was preserved in liver tissue of G-MS (PB) group best viewed with reticulin stain (Fig. 2). Compared to the G-MS group, there was a reduction in ballooning degeneration, microvesicular steatosis, sinusoidal congestion, and dilation. There were very minimal focal hepatic necrotic and acidophilic bodies (Fig. 1). No bridging fibrosis was found, with only few portal triads showing fibrous portal expansion (Fig. 3). Compared to the G-MS group, the G-MS (PB) group showed less features of liver injury. 

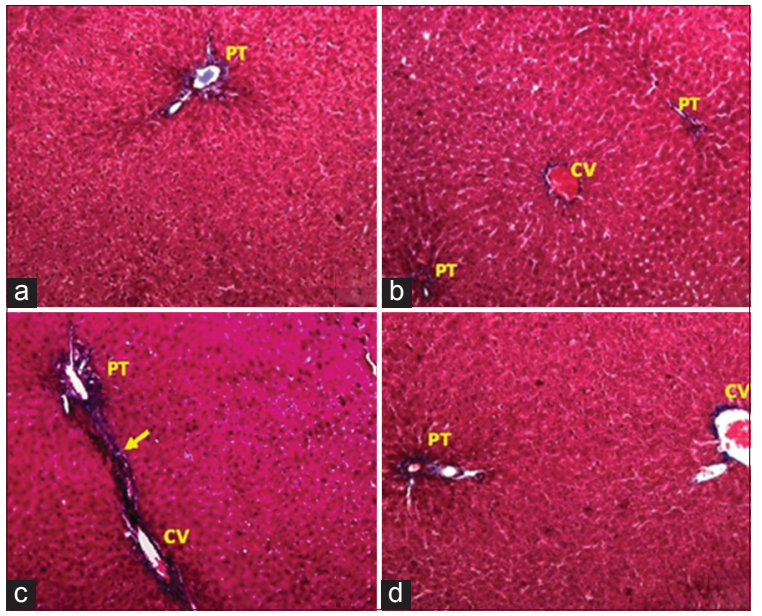

Fig. 3: The G-T80 (a) and G-Piper betle (PB) (b) groups showed no fibrotic changes. (c) The G-Mitragyna speciosa (MS) group showed fibrous portal expansion of the portal tract (PT). There was portal-central bridging fibrosis (black arrow) between the central vein (CV) and PT. (d) The G-MS PB group showed normal distribution of collagen in the PT and CV (Masson's trichrome stain, $\times 10$ )

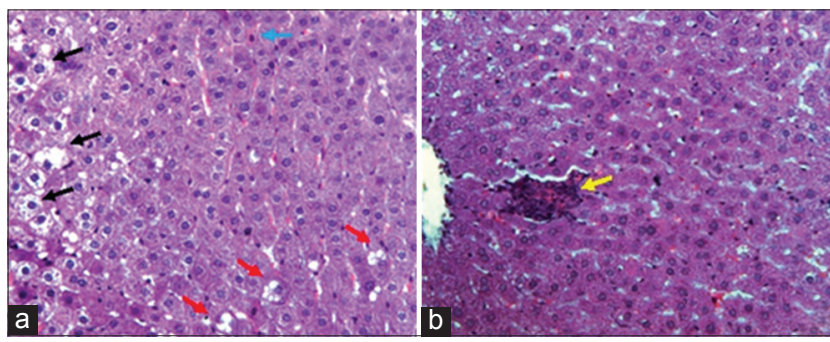

Fig. 4: The liver of the G-Mitragyna speciosa group. Hepatocytes showed ballooning degeneration (black arrow), microvesicular steatosis (red arrow), acidophilic body (blue arrow), and scattered focal necrosis with infiltration of inflammatory cells (yellow arrow). (Hematoxylin and eosin stain, $\times 20$ )

\section{DISCUSSION}

Histopathology assessment is the main method for assessing liver pathology in studies looking at the safety of the new drugs [17]. Histopathology assessment of toxicology studies in experimental animals is needed in predicting toxicity before it can safely proceed to human clinical trials [17]. An earlier histological study was performed using Khat with clove or green tea also showed a protective effect on the liver [18]. Hence, histopathology assessment was used in various toxicological studies in assessing toxicity of new drug or natural product. We also relied on histopathological evidence.

Liver of the G-PB group, which received $200 \mathrm{mg} / \mathrm{kg} /$ day of PBME, had comparable features with the G-T80 group. Earlier study reported administration of PB ethanol extract for 28 days did not exhibit liver injury [16]. Oral administration of methanol extract of PB leaves was reported to be safe with its median lethal dose (LD50) more than $5000 \mathrm{mg} / \mathrm{kg}[19]$.

Acute liver injury can be expressed as cytotoxic injury [4]. Cytotoxic injury may present as steatosis, degeneration, or necrosis [4]. In the G-MS group which received MSME $500 \mathrm{mg} / \mathrm{kg} /$ day, the liver showed microvesicular steatosis,

ballooning degeneration, acidophilic bodies (eosinophilic degeneration), and scattered focal necrosis. There were differences in the pattern of necrosis found in our study compared to earlier studies which observed fatty changes with centrilobular necrosis following a single dose of MS extract $[7,8]$. In the present study, the liver showed scattered focal necrosis with microvesicular steatosis. Liver injury caused by toxic substance had variable histological presentation [4]. Scattered focal necrosis which was classified under non-zonal necrosis was reported to be the most common necrotic pattern of drug-induced liver injury [4]. Focal and non-zonal necrosis was presumed to be caused by apoptosis and nonzonal confluent necrosis may have resulted from severe apoptosis [4]. Acidophilic bodies suggestive of degenerative changes that preceded apoptosis were found scattered in the liver of the G-MS group, which may explain the scattered focal necrosis pattern found in this group.

Sinusoidal congestion was present in the group treated with MSME which was the similar to findings found in the earlier studies which observed the effect of MS extract in acute and subacute administration [7-9]. This feature was also found in liver biopsy of an individual who abused MS and had liver injury [5].

In general, fibrosis is considered as an irreversible consequence of liver damage [20]. Under Masson trichrome stain, areas of fibrous portal expansion and bridging fibrosis were observed in the G-MS group. This suggests that subchronic exposure of MSME may lead to fibrosis formation and had potential to cause irreversible liver damage.

Following concomitant administration of PBME in the G-MS (PB) group, the liver showed less hepatotoxicity features. There was fewer microvesicular steatosis, ballooning degeneration, acidophilic bodies, focal hepatic necrotic, sinusoidal congestion, and dilation with very minimal fibrous portal expansion. PB was reported to possess hepatoprotective action. Studies examined the effect of PB on various toxic compounds including cadmium, carbon-tetrachloride, D-galactosamine, and ethanol which showed PB extract significantly reduced liver injury caused by the toxic compounds [13-16]. PB extract exhibited high antioxidant activities [21]. Active compound isolated from PB including allylpyrocatechol and chavibetol increases radicals scavenging activities [20]. Several mechanisms were postulated to be involved in the development of hepatotoxicity which included increase oxidative stress [22]. Hence, high level of antioxidant in PB may increase radical scavenging activities and this may lead to a reduction of oxidative stress. Thus, administration of PBME may reduce the toxic effect of MS and reduce any injury to the liver.

\section{CONCLUSION}

In the present study, subchronic administration of MSME $500 \mathrm{mg} / \mathrm{kg} / \mathrm{day}$ caused liver injury with fibrosis formation. Concomitant treatment with PBME for 28 days significantly reduced the liver injury induced by MSME. Hence, the present study opens the opportunity for future research to observe the mechanism involved in the hepatoprotective effect of PBME in inhibiting liver injury in abusers with MS ingestion. Future clinical trials are advised to try PB extract as an effective antidote for treating hepatotoxicity.

\section{ACKNOWLEDGMENT}

The authors acknowledge the financial assistance received from grant UKM FF-2014-366 and DLP 2014-009.The authors acknowledge the technical help received from Nur Ahlina Abdullah.

\section{AUTHOR'S CONTRIBUTION}

All the authors contributed equally to planning, conductance ofstudy, interpretation of results and writing.

\section{CONFLICTS OF INTERESTS}

None of the authors have any conflicts of interests to declare.

\section{REFERENCES}

1. Raffa RB, editor. Kratom and Other Mitragynines: The Chemistry and Pharmacology of Opioids from a Non-Opium Source. London: CRC Press; 2014. 
2. Ulbricht C, Costa D, Dao J, Isaac R, Le Blanc YC, Rhoades J, et al. An evidence-based systematic review of Kratom (Mitragyna speciosa) by the natural standard research collaboration. J Diet Suppl 2013;10:152-70.

3. Hassan Z, Muzaimi M, Navaratnam V, Yusoff NH, Suhaimi FW, Vadivelu R, et al. From kratom to mitragynine and its derivatives: Physiological and behavioural effects related to use, abuse, and addiction. Neurosci Biobehav Rev 2013;37:138-51.

4. Zimmerman HJ. Hepatotoxicity: The Adverse Effects of Drugs and other Chemicals on the Liver. Philadelphia, PA: Lippincott Williams \& Wilkins; 1999.

5. Kapp FG, Maurer HH, Auwärter V, Winkelmann M, HermannsClausen M. Intrahepatic cholestasis following abuse of powdered kratom (Mitragyna speciosa). J Med Toxicol 2011;7:227-31.

6. Dorman C, Wong M, Khan A. Cholestatic hepatitis from prolonged kratom use: A case report. Hepatology 2015;61:1086-7.

7. Harizal SN, Mansor SM, Hasnan J, Tharakan JK, Abdullah J. Acute toxicity study of the standardized methanolic extract of Mitragyna speciosa korth in rodent. J Ethnopharmacol 2010;131:404-9.

8. Kamal MS, Ghazali AR, Yahya NA, Wasiman MI, Ismail Z. Acute toxicity study of standardized Mitragyna speciosa korth aqueous extract in sprague-dawley rats. J Plant Stud 2012;1:120.

9. Sakaran R, Othman F, Jantan I, Thent ZC, Das S. Effect of subacute dose of Mitragyna speciosa korth crude extract in female sprague dawley rats. J Med Bioeng 2014:3:98-101.

10. Ilmie MU, Jaafar H, Mansor SM, Abdullah JM. Subchronic toxicity study of standardized methanolic extract of Mitragyna speciosa korth in sprague-dawley rats. Front Neurosci 2015;9:189.

11. Kumar N, Misra P, Dube A, Bhattacharya S, Dikshit M, Ranade S. Piper betle Linn. A maligned pan-asiatic plant with an array of pharmacological activities and prospects for drug discovery. Curr Sci 2010;99:922-33

12. Pradhan D, Suri KA, Pradhan DK, Biswasroy P. Golden heart of the nature: Piper betle L. J Pharm Phytochem 2013;1:147-67.

13. Saravanan R, Prakasam A, Ramesh B, Pugalendi KV. Influence of Piper betle on hepatic marker enzymes and tissue antioxidant status in ethanol-treated wistar rats. J Med Food 2002;5:197-204.

14. Young SC, Wang CJ, Lin JJ, Peng PL, Hsu JL, Chou FP, et al. Protection effect of piper betel leaf extract against carbon tetrachloride-induced liver fibrosis in rats. Arch Toxicol 2007;81:45-55.

15. Pushpavalli G, Veeramani C, Pugalendi KV. Influence of Piper betle on hepatic marker enzymes and tissue antioxidant status in D-galactosamine-induced hepatotoxic rats. J Basic Clin Physiol Pharmacol 2008;19:131-50.

16. Milton Prabu S, Muthumani M, Shagirtha K. Protective effect of Piper betle leaf extract against cadmium-induced oxidative stress and hepatic dysfunction in rats. Saudi J Biol Sci 2012;19:229-39.

17. Greaves P. Histopathology of Preclinical Toxicity Studies: Interpretation and Relevance in Drug Safety Evaluation. London: Academic Press; 2011.

18. Anila R, Sathiya S, Babu CS, Rajkumar J. In vitro and in vivo protective effects of ambrex, a polyherbal formulation, against methotrexate induced damages in hepatic cells. Int J Pharm Pharm Sci 2015;7:164-70.

19. Al-Adhroey AH, Nor ZM, Al-Mekhlafi HM, Amran AA, Mahmud R. Antimalarial activity of methanolic leaf extract of Piper betle L. Molecules 2010;16:107-18.

20. Cotran RS, Kumar V, Collins T, Robbins SL. Robbins Pathologic Basis of Disease. $6^{\text {th }}$ ed. Philadelphia, PA: W.B. Saunders Company; 1999. p. 30-345.

21. Rathee JS, Patro BS, Mula S, Gamre S, Chattopadhyay S. Antioxidant activity of piper betel leaf extract and its constituents. J Agric Food Chem 2006;54:9046-54.

22. Jaeschke H, Gores GJ, Cederbaum AI, Hinson JA, Pessayre D, Lemasters JJ, et al. Mechanisms of hepatotoxicity. Toxicol Sci 2002;65:166-76 